\title{
Avant-propos : L'innovation à l'IUFM de Montpellier
}

\section{Alain Lerouge}

\section{OpenEdition}

1 Journals

Édition électronique

URL : http://journals.openedition.org/trema/2886

DOI : 10.4000/trema.2886

ISSN : 2107-0997

Éditeur

Faculté d'Éducation de l'université de Montpellier

\section{Édition imprimée}

Date de publication : 1 janvier 2003

Pagination : 3-4

ISSN : 1167-315X

\section{Référence électronique}

Alain Lerouge, «Avant-propos : L'innovation à l'IUFM de Montpellier », Tréma [En ligne], 20-21 | 2003 mis en ligne le 01 avril 2003, consulté le 22 septembre 2020. URL : http://journals.openedition.org/ trema/2886 ; DOI : https://doi.org/10.4000/trema.2886

Ce document a été généré automatiquement le 22 septembre 2020.

Trema 


\title{
Avant-propos : L'innovation à l'IUFM de Montpellier
}

\author{
Alain Lerouge
}

1 Depuis la rentrée 2000, l'IUFM de Montpellier encourage la réalisation de projets innovants concernant les formations dispensées aux stagiaires et aux étudiants. Un appel à propositions est lancé en début de chaque année scolaire, et à l'issue de deux années de fonctionnement vingttrois projets ont été réalisés. L'ensemble des documents de synthèse rendant compte de ces projets est édité en cinq fascicules représentant un document de 450 pages dont nous avons tiré ce $\mathrm{N}^{\circ}$ spécial de la revue TREMA.

2 Sur l'ensemble des projets réalisés, seulement quatorze paraissent dans la revue ${ }^{1}$. Il ne faut pas voir dans cette réduction un tri sur des critères de qualité, mais simplement le fait que certains collègues n'ont pas souhaité aller au-delà d'un simple rapport de réalisation, et que d'autres n'ont pu tenir les dates butoir imposées par le calendrier de publication. Je voudrais dire ici mon regret de n'avoir pu tout publier, et rendre hommage au travail fait par l'ensemble des proposants.

3 Je ne serai pas le premier à insister sur la lourdeur de notre système de formation, et sur sa forte résistance au changement... Dans ce contexte institutionnel, toute décision imposée a du mal à prendre effet, et à l'évidence on ne peut diriger un IUFM par injonction descendante. Seul un véritable management participatif, s'appuyant sur la dynamique de projet des acteurs, peut produire du changement en profondeur. Dans ce contexte, la question de l'innovation est véritablement une question forte au sein de nos instituts, et son encouragement devrait être une priorité politique de leur direction.

4 C'est le choix qui a été fait depuis deux ans à l'IUFM de Montpellier, ce $N^{\circ}$ spécial de la revue en témoigne, il en montre toute la richesse et toute la difficulté. 


\section{NOTES}

1. Les textes non publiés sont ceux de :

- Mme ANTOINE Isabelle

Dispositif d'appui aux stagiaires PE2 en grande difficulté

- $\mathrm{M}^{\text {me }}$ BERTHOU Guillemette

Améliorer la formation PLC2 en sciences de la vie et de la terre et sciences physiques - $\mathrm{M}^{\mathrm{me}}$ CHEMLA Marie-Thérèse

Travailler en équipe de formation

- $\mathrm{M}^{\mathrm{me}}$ FERRÉ Danielle

L'avenir s'imagine

- $\mathrm{M}^{\mathrm{me}}$ HERBLOT Martine et M. THYSS Daniel

Les personnages de contes

- M. JEAN Alain

Accompagnement à distance des formations didactiques des PLC2

- $\mathrm{M}^{\mathrm{me}}$ MIRGALET Christine

Partenariats culturels en parcours personnalisés

- $\mathrm{M}^{\text {me }}$ SLEE Brigitte

L'anglais à l'école élémentaire, collaboration avec l'université d'York

- M. SOULÉ Yves

Pour une didactique de l'image

\section{AUTEUR}

\section{ALAIN LEROUGE}

Directeur Adjoint de l'IUFM de Montpellier Chargé de la recherche, l'innovation et la formation de formateurs. 